\title{
Arthur Allen Cohen: ser judío en América
}

Arthur Allen Cohen: Being a Jew in America

Miguel Giménez

mgblunden@hotmail.com

Universidad Pontificia Comillas

España

\section{Resumen}

El artículo pretende ser el análisis de la complejidad de la identidad judía en Estados Unidos y en términos generales, su reivindicación en la llamada "experiencia americana", a través de la obra del conocido autor estadounidense Arthur Allen Cohen, novelista, editor, escritor, filósofo y teólogo norteamericano, conocido especialmente por su obra The Tremendum, una de las interpretaciones más originales del Holocausto del siglo XX. El artículo realizará esto mediante la presentación de su vida y pensamiento.

Palabras clave: Asimilación - Identidad judía - Judío natural y sobrenatural - Teología judía - Holocausto.

\begin{abstract}
This paper is meant to be an analysis of the complexity of Jewish identity in America and in general terms, its vindication of the so-called "American experience" in the works of the American author Arthur Allen Cohen, an American novelist, editor, philosopher and theologian, specially known for his book The Tremendum, one of the most original
\end{abstract}


interpretations of the Holocaust in the 20th Century. The paper means to do so by introducing the main aspects of his life and work.

Key words: Assimilation - Jewish identity - Natural/Supernatural Jew - Jewish Theology Holocaust.

\section{Introducción y sentido del artículo}

Arthur Allen Cohen es un autor poco conocido en el contexto hispano e hispanohablante, pero de enorme prestigio, no sólo por su dimensión como novelista, pero ante todo como teólogo en el contexto americano. Es, junto con Emil Fackenheim, Eliezer Berkovits, Ignaz Maybaum y Richard Rubenstein, una parte de la pléyade de pensadores dedicados a la corriente de lo que ha venido a llamarse Teología del Holocausto, que más que una teología, es una preocupación filosófico-religiosa por la Shoá (Morgan, 2001). Su fino intelecto hicieron de él, un "escritor para escritores" como fue llamado y le convirtió en el autor de una obra que oscila entre la filosofía y la teología, la crítica cultural, el coleccionismo de arte, la bibliofilia, la fascinación por las vanguardias europeas y el "momento americano" del judaísmo, entre otras denominaciones, en las que los planteamientos teológicos y filosóficos se plasman en personajes literarios y en estudios filosófico-teológicos. Cohen habla a través de la filosofía, de la teología, de la ficción y al modo de los diálogos platónicos, donde cada personaje expresa un prisma, una versión, una faceta de la identidad judía.

Cohen manifiesta su identidad judía a través de una creatividad enorme y una incesante obra diversa, mostrando la complejidad y la heterogeneidad de ser judío en la época moderna. Fuera en la Rusia de Stalin o en la América de los años sesenta, se trataba de mostrar que ser judío no es meramente la adhesión a una serie de normas éticas y comportamientos ordenados por mandamientos estrictos, sino fundamentalmente, una actitud vital que define un grupo de personas de origen diverso, de culturas distantes, pero cuyo rasgo esencial y definitorio es reconocerse como pertenecientes al Pueblo Escogido. 
Esta actitud en el caso concreto de Cohen, se manifestó de las más diversas formas, permeando su vida y su obra escrita.

Arthur Allen Cohen (1928-1986) es uno de aquellos individuos cuyo abanico de intereses superan con creces los límites de una vocación única, abarcando la teología, la filosofia, el coleccionismo de arte y las vanguardias rusas, las que compartió con su mujer Elaine Lustig-Cohen. Supo condensar una multitud de tendencias y se sentía tan a gusto hablando de la dimensión sobrenatural del Pueblo Judío como de la abstracción geométrica de Sonia Delaunay ${ }^{1}$.

Arthur Allen Cohen es una de las figuras más interesantes y menos conocidas del panorama del pensamiento judío contemporáneo. Pertenece a ese linaje de pensadores que a medio camino entre la teología y la literatura, entre el pasado y el presente, renueva el debate intelectual no solo estadounidense, ofreciendo un estudio serio de la tradición filosófica y teológica del judaísmo europeo y norteamericano, a la vez que una visión de las relaciones con el Cristianismo; siendo, por ejemplo, su labor como editor, una exposición y presentación para el mundo anglosajón de algunas de las dificultades intelectuales tras la Segunda Guerra Mundial. Hemos querido sintetizar las complejidades de su obra en este artículo. El contenido del mismo consistirá en un esbozo biográfico, seguido de una división temática de su obra, que permita estimular el interés en su obra y en su particular visión del judaísmo.

\section{Esbozo biográfico}

Arthur Allen Cohen nace en Nueva York en $1928^{2}$. De un medio asimilado, Cohen entra en la universidad a la temprana edad de 16 años. Llega a tener una especie de crisis espiritual en los albores del año 1945, planteándose incluso convertirse al cristianismo. Sus padres en

\footnotetext{
${ }^{1}$ Delaunay, Sonia (1885-1979), una de las artistas francesas más renombradas de su época, conocida por la abstracción geométrica, concepto artístico que la caracterizó y que supo aplicar a muchos de sus diseños de muebles y ropa, entre otros campos (Cf. Britannica. Global Edition, 2009, pág. 4454-4455).

${ }^{2}$ Los datos biográficos están tomados de las siguientes fuentes: Mendes-Flohr y Stern, 1998, p.11-25; Giménez Blunden, 2012, p.88-90; Shatzky y Taub, 1997, p.46-47; Cronin y Berger, 2009, p.52.
} 
vez de acudir a un psicólogo, acuden al rabino Milton Steinberg ${ }^{3}$, una de las mentes más lúcidas del judaísmo de ese momento. La tutela espiritual de este rabino lleva a Cohen a conocer de forma más profunda sus raíces, embarcándole en el estudio del hebreo y haciéndole tomar conciencia de su propia tradición e identidad judía. Este viraje hacia el judaísmo estará condicionado permanentemente a lo largo de toda su vida por la omnipresencia del Cristianismo, como punto de referencia desde el que construir su propia visión y toda su vida, por tanto optará por un judaísmo que no "hereda", sino que es fruto de una "elección" consciente. (Mendes-Flohr y Stern, 1998, p.32-41)

En 1946 se gradúa y en 1949 obtiene el máster con una tesina sobre el concepto de la paradoja en Kierkegaard y Nietzsche, abandonando posteriormente sus estudios de doctorado siendo el título de la tesis El uso de la metáfora y el lenguaje metafísico en la literatura. Arthur Allen Cohen pasa la segunda mitad de 1949 en la Universidad Hebrea de Jerusalén estudiando hebreo y trabándose la amistad de pensadores de la talla de Martin Buber, Ernst Simon y Hugo Bergmann. Tras su estancia en Jerusalén, prosigue estudios en el Jewish Theological Seminary de Nueva York para estudiar filosofía judía medieval, dejando dicho seminario en 1951. El espíritu de Cohen es libre y no se integra en los núcleos organizados del judaísmo tradicional. No quiere verse condicionado ni por prejuicios ni opiniones ajenas. Quien se había dicho que era el Maimónides de su generación, repartirá su vida entre el mundo del libro y el mundo de la ciencia de Dios.

Se casa en 1956 con Elaine Lustig, viuda de Alvin Lustig, el diseñador gráfico, con quien inicialmente le unió una relación comercial y que acabó en historia de amor. Fruto de su matrimonio, nacería una sola hija, Tamar Judith, que seguiría la prestigiosa carrera como diseñadora gráfica de su madre.

\footnotetext{
${ }^{3}$ Steinberg, Milton (1903-1950), rabino, filósofo, formado en Nueva York, doctorado en la Universidad de Columbia (EE.UU.) en 1924, fue uno de los teólogos judíos más destacados de su época (Cf. Mendes-Flohr y Stern, 1998, p.13).

4 Tomado del artículo de 1959 de Cohen: Cf. Cohen, A. (1959). Why I choose to be a Jew. Harper's Magazine, 218 (1307), 63-66.
} 
El mundo editorial será su fuente primordial de ingresos y satisfacción, aunque su pasión fundamental sea la teología. Funda la editorial Noonday Press junto con el poeta Cecil Hemley. Su éxito es tal que cuatro años más tarde funda otra, la Meridian Books en 1954. En 1960 pasa ser el director de literatura religiosa de Holt, Rinehart and Winston, que por aquel entonces, era seguramente uno de los sellos editoriales de mayor importancia en Estados Unidos. Sus grandes obras de teología se escriben a lo largo del periodo de 1951 a 1969. Durante 18 años (1951-1969), combina a partes iguales, su pasión por el mundo editorial y la teología. En el año 1973, funda junto a su mujer Elaine ${ }^{5}$, una tienda de objetos antiguos, especializada en libros de arte llamada Ex Libris, cuya especialidad era el arte moderno, sobre todo las vanguardias rusas, y se mantuvo en funcionamiento hasta 1998. Fue, con toda seguridad, el período más feliz de su vida. Por desgracia, una leucemia apagó prematuramente su vida en 1986. En la actualidad, sus manuscritos y pruebas de imprenta se encuentran en la Beinecke Library de manuscritos raros de la Universidad de Yale, en Estados Unidos, y otros textos inéditos están en la propiedad de su viuda Elaine.

\section{Obra de Arthur Allen Cohen. Introducción}

La obra de Arthur Allen Cohen tiene múltiples facetas: una teológico-filosófica, una novelística y una artística, que coinciden cronológica y temáticamente con momentos muy diversos de su biografía. ${ }^{6}$

Primordialmente, Arthur Allen Cohen está considerado dentro de la llamada Teología del Holocausto, como miembro de la corriente de pensadores que tiene como reflexión central la Shoá (Cohen, 1963). Sin embargo, su desarrollo intelectual sui generis y los géneros literarios y temáticas que aborda hacen de él un pensador singular. Cohen tiene varios registros y emplea distintos medios literarios para reflejar la tensión interna de su identidad judía. En primer lugar, tenemos al Cohen teólogo. También tenemos al Cohen novelista, siendo su obra de ficción, continuidad de los temas teológicos e intelectuales mediante la ficción narrativa. Otra faceta es la del Cohen editor, que aborda la actualidad y la tradición

\footnotetext{
${ }^{5}$ Lustig-Cohen, Elaine (1927-), diseñadora gráfica de enorme prestigio en Estados Unidos, que además de ser su mujer, diseñaba las portadas de los libros que él publicaba, y de algunas de las obras de las que él era autor. ${ }^{6}$ Estos núcleos definen su obra de forma esencial.
} 
filosófica de forma inaudita, siendo tanto la presentación como el contenido de su obra, de primer orden. En cuarto lugar, tenemos al Cohen bibliófilo, historiador del arte y coleccionista, abarcando la representación de la Biblia en el arte cristiano, judío e islámico (Cohen, 1963); la obra de Herbert Bayer, el diseñador gráfico y publicista de la Escuela de la Bauhaus (Cohen, 1984); una representación fotográfica con texto de Cohen de la vida de la comunidad jasídica ${ }^{7}$ en Estados Unidos (Cohen, 1970); el artista Jacob Steinhardt (Cohen, 1987), la abstracción geométrica de Sonia Delaunay (Cohen, 1984) y sus colecciones de arte vanguardista (Cohen, 1973).

\section{Obra teológica y filosófica}

En Cohen, donde empieza lo teológico y acaba lo filosófico resulta casi una distinción inviable. Varias son las obras en las que aborda los temas que le preocupan esencialmente acerca de esta temática. Cinco son las que componen esta parte de su reflexión filosófica, tres menores y dos fundamentales.

Entre los menores están: Martin Buber, de 1957; La anatomía de la fe: Ensayos teológicos de Milton Steinberg, de 1960; El mito de la tradición judeocristiana y otros ensayos disidentes, de 1971, y ¿Si no ahora, cuándo?, escrita en 1973, donde mantiene un diálogo con el rabino Mordecai Kaplan.

Sus dos obras más conocidas son esencialmente El judio natural y el judio sobrenatural, de 1967, y El Tremendum, su obra teológica mayor, publicada en $1981^{8}$. Empezaremos pues, por orden inverso, por lo más esencial y conocido antes, para adentrarnos luego en los demás textos. El teólogo Cohen se manifiesta con radical contundencia en El judío natural y el sobrenatural, pero ante todo en su libro teológico mayor, que agrupa los campos de concentración y lo que sucedió allí. Cohen aporta con este concepto, un vocabulario que empieza a esgrimirse en los años sesenta, donde tímidamente y después de quince años de

\footnotetext{
${ }^{7}$ Movimiento religioso judío, que se origina en el siglo XVIII, que casi desaparece en el Holocausto, y cuya comunidad estadounidense es uno de los pilares de su supervivencia y continuidad, motivo por el que cobra especial importancia la obra testimonial de Cohen.

${ }^{8}$ Mantenemos la mayúscula en su concepto de Tremendum a lo largo del texto, como del término Holocausto (Nota del autor).
} 
haber finalizado la segunda conflagración mundial, se empieza a pensar y a discutir la Catástrofe.

El Tremendum. Una interpretación teológica del Holocausto fue el fruto de una serie de cuatro conferencias que imparte entre 1974 y 1979, donde intenta explicar la Shoá desde un punto de vista fenomenológico, teológico y filosófico. Es uno de los grandes intentos de dar sentido, significación y fundamento a dicha realidad. El término empleado que procede del Mysterium Tremendum, de Rudolf Otto, es el intento de expresar todo cuanto pasó en los campos de concentración y mostrar lo inefable, comprender lo incomprensible, hablar de lo que solamente suscita silencio. Cohen es uno de los más destacados pensadores del siglo $\mathrm{XX}$ que se atreve a hablar de lo indecible y que intenta crear un aparato categorial específico, que reflexione sobre la Shoá.

Hablar del Holocausto, sin embargo, tiene una dimensión mucho más amplia y, en el fondo, en Cohen se traduce como la reflexión sobre el mal en el mundo. Este nuevo término, este novum, se introduce en el vocabulario filosófico y teológico del siglo XX como sus otros sinónimos, como Catástrofe, exterminio y "evacuación a Theresienstadt", entre otros. El Tremendum se alza como el concepto abanderado del único universalismo que ha persistido de forma perenne a nivel histórico. No es el judío en su particularidad el perseguido, el señalado, sino el "Judío" universal. Los descendientes de Abraham son, en su persecución, "los escogidos, sin posibilidad de remisión, de forma extrema y total" (Cohen, 1981). Desde un punto de vista fenomenológico, Cohen crea un decálogo que caracteriza a dicho concepto, cuyos pilares fundamentales son:

1) El Tremendum queda fuera del orden histórico, tiene hambre de ser, del que se percibe más el mal que el bien.

2) Frente a la unicidad de la Catástrofe planteada por autores como Emil Fackenheim, el Tremendum es un universal como muchos ha habido a lo largo de la historia reciente; el esfuerzo por singularizar el fenómeno permite precisamente el silencio, no permite hablar del tema. 
3) El mal tiene una realidad substancial, el mal tiene sed de ser y se ha manifestado y encarnado históricamente.

4) La nueva "teodicea" coheniana parte de la base de la predominancia del mal en el mundo actual.

5) Pensar en el Tremendum hace hincapié en los grandes temas: la realidad del mal y la existencia de Dios, los extremos de la maldad y la libertad del hombre y la presencia del mal frente al poder de Dios.

6) El lenguaje no es capaz de expresar esta realidad histórica y se nos presenta como un concepto aporético, contradictorio, que no puede ser absorbida por su significación en la narrativa histórica.

7) La dimensión ontológica del Tremendum no puede limitarse mediante las tradicionales categorías históricas, poco adecuadas y de poca importancia frente a acontecimientos de tal envergadura.

8) Nunca antes había existido un horror a tal escala, de modo que es inconcebible que los primeros sistemas filosóficos de la modernidad (Hegel y Marx) pudieran ser paradigmas suficientemente omnicomprensivos como para valorar esta nueva realidad.

9) Los paradigmas de la modernidad, el optimismo, la confianza en el progreso y el liberalismo se han puesto en tela de juicio al abordar esta cuestión. Un acontecimiento tal supuso la ruptura de relaciones personales y sociales, la imposibilidad de los judíos de poder aspirar a los sueños del progreso y la emancipación que las corrientes liberales de Europa preconizaban.

10) El Tremendum se caracteriza por su resonancia histórica. No queda acotado meramente al periodo histórico en el que se desarrolló, sino que reverbera, de modo que sus consecuencias, significación y proceso perviven no sólo como recuerdo, sino como modelo de ulteriores barbaries.

Hay algo, sin embargo, en la percepción del Tremendum que es distinto para el judío y el gentil. El Tremendum (a pesar del judío) forma una unidad indisoluble con su realidad. 
Para el no judío, es objetivo, un hecho externo a su propia realidad; para el judío, resulta indisoluble, esencial, inmanente, constitutivo de su misma identidad.

La otra gran obra de Cohen es El Judio Natural y Sobrenatural. En ella define su propia noción del concepto de lo teológico y distingue en una idea que no es original de él, la diferencia existente entre el judío natural y sobrenatural, imprimiendo su visión de la doble naturaleza de la identidad judía, una horizontal en su relación con la historia y los demás pueblos de la Humanidad, y una vertical, en su particular Alianza con Dios.

La obra, dedicada a sus "judíos naturales”, su mujer Elaine y su hija Tamar Judith, contiene cuatro capítulos, donde analiza, en una primera parte, el proceso del pensamiento desde finales del siglo XV hasta el XX. Su primer capítulo se denomina El siglo de la incertidumbre (desde Solomon Ibn Berga, pasando por Moses Mendelssohn, Nachman Krochmal, Leopold Zunz, Heinrich Graetz, Samson Raphael Hirsch, Moses Hess, Ahad Ha-Am, Simon Dubnow y Chaim Nachman Bialik).

Para seguir, en una segunda parte, con el llamado Renacimiento Judeo Germano, con Hermann Cohen, Leo Baeck, Franz Rosenzweig y Martin Buber. El capítulo tercero incide en el llamado "momento americano" del judaísmo, esta particular manifestación del mismo que tiene su expresión en una nueva forma de entender el exilio y la diáspora, en autores como su profesor Mordecai M. Kaplan, su maestro Milton Steinberg, Abraham Joshua Heschel y Will Herberg. La unión de lo natural y lo sobrenatural encarnado en el judío concreto recorre la cuarta parte del libro, donde Cohen da rienda suelta a su metafísica de la historia, a la teología como la ciencia de la historia sagrada, al siempre complejo concepto del mesianismo y a la renovación de la vocación de ser judío.

Las otras cuatro obras se ciñen a ejemplos concretos del pensamiento judío, a personajes específicos y sus aportes. De Martin Buber destaca la esencia de su filosofía, la relación dialógica Yo-Tú, la importancia del jasidismo y cómo debe desenvolverse el hombre en el mundo. Cohen no podía menos que hacerle un homenaje a su maestro Milton Steinberg, en 1960, publicándole sus escritos teológicos en La Anatomía de la fe. 
En El Mito de la tradición judeocristiana, obra dedicada a sus padres, desenvuelve la tensión perenne entre ambas comunidades, siendo que no se llega jamás a una solución real y a una verdadera conciliación. Se abordan pues, los fundamentos y definiciones teológicohistóricas de esta relación, las polémicas y disputas tradicionales (especialmente centrado en el tema de la conversión y la figura de Jesús). Asimismo, se trata la actualidad y las posibilidades de un diálogo sincero, que tiene la especial dificultad de moverse en medio de las convulsiones del silencio del Holocausto y la creciente ola de secularización y humanismo que invadía el panorama cultural occidental en la década de los años sesenta y setenta del siglo $\mathrm{XX}$.

De ¿Si no ahora, cuándo?, sólo cabe decir que es el resultado de analizar el surgimiento de una nueva identidad judía, fundada en la renovación planteada por el reconstruccionismo, representado por Mordecai Kaplan, cuestionada por Cohen en una serie de charlas que mantuvieron entre el 10 de septiembre y el 7 de octubre de 1971.

\section{Obra novelística}

Dos expertos en la obra de Cohen, afirman con total razón, que hay dos tendencias fundamentales en su obra novelística. La primera, que afirma que lo que dice en la ficción, son de hecho, reflexiones de orden teológico (Cronin y Berger, 2009) y otra que sus textos son la forma americana de la expresión de la identidad judía, la que siempre se había planteado desde una óptica germana o fundamentalmente europea, o en palabras de Claire R. Satlof, citando a Cynthia Ozick, "Arthur Cohen es el primer escritor de cualquier generación americana en componer una ficción profundamente judía basada en una profunda temática occidental" (Shatsky y Taub,1997, p.51-52).

El mismo Cohen no distingue entre dos hilos narrativos, uno teológico y otro novelístico, como una unidad total y absoluta de intereses, mantenidos a flote por un delicado equilibrio: "Mantengo la unidad de ambos cuerpos de trabajo (...) no como la suspensión azarosa de partículas en un campo magnético, sino que cual un imán humano, teniendo 
voluntad e inteligencia, puedo determinar eficientemente lo que exprese y cuanto debo suprimir". (Mendes-Flohr y Stern, 1998, p.257 y p.421)

A lo largo de cinco novelas, una de las producciones más singulares de Cohen, analiza, expresa y muestra con inigualable estilo literario, la continuación de su pasión teológica. Las novelas hay que leerlas como quien analiza lo que viene a llamarse una novela de tesis, la expresión en ficción literaria de lo que son profundas convicciones filosóficas, teológicas e identitarias.

De este modo en la obra En los días de Simon Stern, de 1973, nos presenta a un Segundo Mesías que, presente en los barrios de Nueva York, se propone rescatar en la vieja Europa, a los supervivientes de la barbarie nazi. En su obra de 1976, Un héroe de su tiempo, un poeta judío ruso ubicado también en esa ciudad estadounidense, se ve perseguido por el KGB y se analiza la tensión entre la política y la creatividad artística. En su texto de 1980, Actos de hurto, se recrea con la idea de un ladrón de piezas precolombinas. Y en Una mujer admirable, de 1983, se aprecia la figura de una mujer judía, parecida según expertos a Hannah Arendt, que abandona Alemania, se radica en París y Nueva York, que vive la tensión espiritual de quien precisa un espíritu divino si hemos de vivir una vida con significado (Shatsky y Taub, 1997). En su obra póstuma de 1987, Artistas y enemigos, a lo largo de tres relatos narrativos, recoge su interés por la historia rusa y por las vanguardias artísticas, recreándose en una de sus pasiones intelectuales.

Pero es ciertamente en Los años del carpintero, de 1967, en la primera de sus novelas, donde mejor puede entenderse la tensión existente en su interior, que tiene efectivamente el matiz autobiográfico, que resultaba de su intento de conversión de juventud y, por ende, del cuestionamiento de su propia identidad judía. El texto refleja un conflicto padre-hijo, que resulta de la asimilación y conversión del padre frente al acérrimo judaísmo del hijo. Todos los personajes que salen a lo largo de la pieza literaria tienen en cierto modo, la función de arquetipo, una polifonía de voces al modo de los diálogos platónicos, donde cada personaje muestra una voz distinta, una caracterización. Cohen pone en el asador sus convicciones, su pensamiento, su forma de entender la vida en estos ejercicios literarios, que no son de modo 
alguno reflejo menor ni limitación de su capacidad para especular y para mostrar su pasión teológica.

\section{Crítica y divulgación cultural}

Uno de sus aportes más destacados son algunas de las compilaciones o antologías que realizaba (Cohen, 1970, 1980), como asimismo los escritos concretos o bien diccionarios, en los que editando con otros expertos, los mejores especialistas en teología y filosofía, daban sus definiciones sobre términos de enorme importancia teológica y filosófica. ${ }^{9}$

Como crítico cultural describe curiosamente la aportación de Mao Tse-Tung (Cohen, 1966), diez años antes de su fallecimiento en 1976, donde reflexiona a lo largo de seis capítulos (que incluyen el análisis de sus aportaciones como filósofo, revolucionario, su noción de Estado y la realidad de una sociedad socialista), la obra del máximo dirigente chino, que llevó al enorme gigante asiático a su actual forma política.

Su preocupación por la formación humanística y su relación con la civilización occidental le lleva a la obra colectiva de 1964, donde los valores democráticos de Occidente y el pasado y el presente de las humanidades se analizan con detenimiento (Cohen, 1964). Su interés por la realidad rusa y, con frecuencia, la dificultad que los rusos judíos padecían, se plasma en su texto sobre el malogrado poeta judío ruso Osip Mandelstam (1891-1938) (Cohen, 1974).

\section{Obra artística. El teólogo y el artista}

La pasión de Arthur Allen Cohen por todo lo artístico se traduce primordialmente en seis obras, dos de ellas de una extensión y un tamaño considerables, y las otras breves que puntualizan e incluso muestran de forma física, algunas de las preferencias editoriales del autor. La dos más grandes son las que dedica a Herbert Bayer y Sonia Delaunay, mastodónticos ejercicios, no sólo por la exposición gráfica de la obra, sino también por la interpretación, lectura y comentario de la misma.

\footnotetext{
${ }^{9}$ Cohen, Arthur A. y Mendes-Flohr, P. (1987); Halverson, Marvin y Cohen (1962).
} 
Las de menor formato son La Biblia Hebrea en el Arte Cristiano, Judío y Musulmán (1963); La vía negativa: Una colaboración (1964); Los Delaunays, Apollinaire y Cendrars (1972), El Libro al desnudo (1973) y la obra póstuma El Steinhardt Desconocido (1987).

En la primera obra, más que la importancia del texto bíblico, se trata de ver en qué medida el Libro de los Libros (en su formulación de Pentateuco, los libros de los Profetas, los libros sapienciales y porciones de los apócrifos) ha tenido su influencia en la imaginación creativa de los "mundos cristiano, judío y musulmán". El catálogo de la exposición contiene 120 obras, todas ellas inspiradas en episodios tomados de la Creación, de Adán y Eva, del Arca de Noé, de Abraham y Agar, la concubina egipcia de Abraham, del sacrificio de Isaac, de la lucha de Jacob, de José, de Moisés y la zarza ardiendo, el becerro de oro, los Diez Mandamientos, de Sansón y Dalila, los reyes David y Salomón, Jonás y la ballena, la visión de Ezequiel, los profetas Elías e Isaías, Esther, Daniel, Tobías y finalmente Judith con la cabeza de Holofernes.

Varios grabados y pinturas en cada caso componen la muestra que tuvo lugar en el Museo Judío de Nueva York, del 18 de febrero al 24 de marzo de 1963. Las obras expuestas provenían de galerías y colecciones particulares en su mayoría de Estados Unidos, con notables excepciones, procediendo una de la colección privada del matrimonio Cohen. La exhibición sigue la tendencia habitual de Cohen. El diseño de la portada del certamen fue realizada por Elaine, donde se recrea simplemente el nombre de la exposición y los primeros versos del Génesis en letras color naranja, frente a un fondo blanco, con el nombre de la muestra en negrita. La imagen es importante, pero no menos las palabras de Cohen. Él es quien pone el texto, con frecuencia en papel de distinto color y textura a la de la publicación.

La introducción de Cohen es todo un alegato, una justificación de la colección donde se ponen sobre la mesa, la relación entre el artista y el texto sagrado, o mejor dicho, en qué medida, la obra artística refleja de modos muy diversos, el imaginario y la simbología de los textos sagrados. De los conceptos más simples, surgen grandes obras de arte. Lo 
universal y lo particular se funden en una tradición, que luego tendrá sus ramificaciones en el cristianismo y el judaísmo. La orientación de la exposición es teológica. Lo que resulta importante es el sentido teológico que le imprime a toda la exposición. El sentido del arte para el teólogo Cohen (1967) es “(...) el medio por el que puede pensar más claramente, entender de modo más profundo y amar de forma más ardiente al Dios en el que cree”.

El distanciamiento entre ambas visiones, la teológica y la artística, tiene en el momento actual, su momento álgido. Ambas son formas que muestran el entendimiento del universo por parte del hombre y no deben ser separadas por este motivo. La conciencia histórica y el pensamiento de épocas, quedan cristalizados en el ritmo, movimiento, forma y material de las obras artísticas. Cada forma de arte es, en sí misma, una visión metafísica de la realidad. El teólogo y el artista se unen para comunicar la historia de la búsqueda del significado de la existencia de forma conjunta.

Esta simbiosis entre el teólogo y el artista es una idea muy antigua pero a la vez, de una contemporaneidad exquisita. Hay un correlato entre el iconoclasmo y la iconomaquia, entre el rechazo de la imagen y su intensidad, que se ha mantenido a lo largo de la historia. La tensión fundamental es aquella que se deriva de la separación o de la relación entre lo bello y lo sagrado. Lo irónico del caso, es que cuando lo sagrado tiene su mejor manifestación, casi siempre lo hace en detrimento de la cualidad de la belleza. La iconografía de la religiosidad popular huye del perfeccionismo del arte, intenta mostrar en toda su desnudez, los elementos más singulares de la devoción popular. Y es que el artista primitivo, no el actual, pretende de forma rudimentaria, manifestar, expresar, poner de manifiesto, la existencia, el poder y la realidad de aquello que representa, en su dimensión natural y sobrenatural. La obra de arte adquiere su importancia en tanto que encarna de forma contundente, lo sagrado. Sin embargo, esta diferenciación entre el arte y la religión no está en la actualidad, ya que el arte debe superar los problemas que le acechan en el presente, como son el individualismo, la especialización científica y la creciente secularización de la cultura occidental. 
La Vía negativa de 1964 fue una obra editada de forma privada por Cohen, en colaboración con el pintor abstracto, educador y conferenciante norteamericano Paul Brach (1924-2007). La edición contenía doce copias de placas litografiadas por dicho artista. La edición del libro corrió a cargo de la llamada Tamarind Lithography Workshop, un taller fundado en 1960 por June Wayne, con sede en Los Ángeles. Dicha institución forma parte ahora de la Universidad de Nuevo Méjico, en dicho estado norteamericano y en la ciudad de Alburquerque. Cohen da rienda suelta a este breve libro (no tendrá más de 12 páginas) con doce litografías que simbolizan a Dios o a el Uno de forma abstracta. Cohen tiene la posibilidad de mostrar su faceta más poética en unos versos, que ejemplifican la fusión entre arte y poesía, de los que reproducimos una parte a continuación:

La vía negativa

El Uno era sí mismo,

Único y solitario

El Uno por su propia magnitud,

Se superó a sí mismo,

Escogió forzar fuera los muchos,

Para entenderse a sí mismo.

Cada determinación es una negación.

Lo indiferenciado se determina por negación,

Siendo obligado a ser diferente,

Cesando ser parecido a algo;

Negando la multiplicidad,

Con tal de afirmarse a sí mismo,

Para convertirse en único,

Único para convertirse en lo Uno. 
(Mendes-Flohr y Stern, 1998, p.55).

Los Delaunays, Apollinaire y Cendrars (1972) es un libreto curioso, publicado por The Cooper Union of Art and Architecture, fruto de una conferencia que impartió Cohen el 9 de febrero de 1972, en esta escuela de arte y arquitectura, con sede en Nueva York. Se publicó una edición limitada de 1.000 ejemplares, siendo la decimonónica tipografía Caledonia la empleada, el papel de marca de agua Beckett de Hamilton (Ohio, EE.UU.) y el diseño del libro corriendo a cargo de George Sadek y Susan Mayer. El primero, profesor emérito y decano de esta escuela y la segunda, diseñadora gráfica formada en dicha institucion. Esto refleja el esmero con el que las obras de Arthur Allen Cohen se presentaban a un público expectante. En esta muestra analiza de una forma cultísima, la relación entre tres artistas de singular andadura: Guillaume Apollinaire (1880-1918), el matrimonio formado por Robert Delaunay (1885-1941) y su mujer Sonia, y Blaise Cendrars (1887-1961). El primero y el último son poetas, y el matrimonio son artistas.

El delicado equilibrio entre arte y poesía, y la relación con las vanguardias del siglo XX forman parte del encanto de esta breve, intensa y culta conferencia. Cohen muestra una erudición increíble, moviéndose con una comodidad pasmosa, entre los detalles de la vida y la fecundación intelectual mutua de estas grandes figuras de vanguardia. El primero es un apologeta del cubismo, miembro del movimiento dadaísta e inventor del término surrealismo. El matrimonio Delaunay son los fundadores del movimiento artístico llamado orfismo, cuyo uso de formas geométricas y colores que resaltan son sus características fundamental y, finalmente, Blaise Cendrars, es un poeta que se inserta en el modernismo europeo del siglo XX. Lo visual y lo escrito, la imagen y la palabra tienen especial relevancia para estos autores y es ese intento de equilibrio y de relación fecunda lo que Cohen intenta reflejar. Al final de la obra, a modo de anhelo significativo y contrastando la situación actual del artista y la utopía que vivieron los tres, afirma de su particular relación:

Los pintores de nuestros días, superados por la excelencia de sus dones y la inefectividad social de su arte, se sumergieron (no sin su connivencia activa) en la 
búsqueda de cualquier artefacto de riqueza que pueda ser concebido como sensibilidad contraria al arte y a cualquier humanidad, y están atrapados por la anomía, la desesperación y las locuras varias de nuestros tiempos. Sin embargo, la comunidad de París en las primeras tres décadas de este siglo fue fundada en la convicción de la inutilidad del arte y que no debía resultar en provecho económico, sino que debía ser absolutamente la única cosa digna de realización, que los artistas eran los mejores y los más apasionados de todos los hombres, que los quejicas y narcisistas debían ser aleccionados o puestos a trabajar, y que cuando el poeta acabara el poema y el artista acabara pintando el cuadro, los pintores leerían los poemas y los poetas verían los cuadros y sólo entonces, una conversación humana podría llevarse a cabo. ¿Qué revolución tan civilizada la suya! (Cohen, 1972, p.15-16).

\section{Conclusión}

Arthur Allen Cohen es fruto de la asimilación o del momento norteamericano, que está sediento de la herencia cultural de sus antepasados, sin renunciar a su vida integrada en el sueño americano, enriqueciendo dicha visión con la elección consciente de su vocación judía. Arthur Allen Cohen no se ve obligado precisamente por su "americanismo" a claudicar ante la posible cerrazón y hermetismo de la cultura que abraza. Su independencia intelectual de las instituciones en las que hubiera podido ejercer magisterio le da la libertad suficiente como para poder manejar bien la libertad de alguien que busca en fuentes muy diversas cómo plasmar su vocación judía.

Arthur Allen Cohen, al modo de los hombres del Renacimiento, es un ser humano que calificaríamos en la actualidad de multidisciplinar, que se siente cómodo en varios registros lingüísticos y literarios. Es editor, novelista, se dedica a los negocios, es coleccionista de arte y de libros antiguos, tiene una galería de arte, pero su profundo sentir es el de un teólogo, imprimiendo dicha preocupación a toda su amplia gama de intereses. Sirva este 
artículo pues, para confirmar y realzar la figura de uno que ha significado tanto para el judaísmo americano y que ha mostrado con su vida y obra, la experiencia de ser judío en América. 


\section{Bibliografía}

Cohen, A. (1957). Martin Buber. London: Bowes \& Bowes.

Cohen, A. (1960). The Anatomy of Faith: Theological Essays of Milton Steinberg. California: Harcourt Brace.

Cohen, A. (1963). The Hebrew Bible in Christian, Jewish and Muslim Art. New York: The Jewish Museum.

Cohen, A. (Ed.) (1964). Humanistic education and Western civilization. New York: Reinhart and Winston.

Cohen, A. (1966). The Communism of Mao Tse-tung. Chicago: University of Chicago Press.

Cohen, A. (1967). The Natural and the Supernatural Jew. An Historical and Theological Introduction. London: Vallentine-Mitchell.

Cohen, A. (1967). The Carpenter Years. New York: The New American Library.

Cohen, A. (1970) (Selected with Introductory Essays). Arguments and Doctrines. A Reader of Jewish Thinking in the Aftermath of the Holocaust. New York: Evanston and London, Harper \& Row Publishers.

Cohen, A. (1970) (Texto) People Apart. Hasidism in America. Photographs by Philip Garvin. New York: E.P. Dutton \& Co. Inc.

Cohen, A. (1971). The myth of the Judeo-Christian Tradition and other Dissenting Essays. New York: Schocken Books. 
Cohen, A. (1972). The Delaunays, Apollinaire and Cendrars. New York: The Cooper Union School of Art and Architecture.

Cohen, A. (1973). If not now, when? Toward a Reconstitution of the Jewish People. Conversations between Mordecai M. Kaplan and Arthur A. Cohen. New York: Schocken Books.

Cohen, A. (1973). In the Days of Simon Stern. New York: Random House.

Cohen, A. (1973). The Book Stripped Bare. A Survey of Books by 20th Century Artists and Writers. Long Island/Nueva York.

Cohen, A. (1974). Osip Emilievich Mandelstam. An Essay in Antiphon. Michigan: Ardis.

Cohen, A. (1976). A Hero in His Time. Chicago and London: The University of Chicago Press.

Cohen, A. (1980). Acts of Theft. Chicago and London: The University of Chicago Press.

Cohen, A. (1980). Selected, edited and introduced. The Jew. Essays from Martin Buber's Journal Der Jude, 1916-1928 Translated from the German by Joachim Neugroschel. Alabama: The University of Alabama Press.

Cohen, A. (1981). The Tremendum. A Theological Interpretation of the Holocaust. New York: Crossroad.

Cohen, A. (1983). An Admirable Woman. Boston: David R. Gordine Publisher.

Cohen, A. (1984) (Texto) Sonia Delaunay. New York, Harry N. Abrams Inc. 
Cohen, A. (1984). Herbert Bayer. The Complete Work. Cambridge-Massachusetts/LondonEngland: The MIT Press.

Cohen, A. y Mendes-Flohr, P. (Ed.) (1987). Artists and Enemies. Three Novellas. Massachussets: David R. Gordine Publisher.

Cohen, A. (1987). The Unknown Steinhardt. New York: The Jewish Museum.

Cohen, A. (1988). Contemporary Jewish Religious Thought. Original Essays on Critical Concepts, Movements and Beliefs. New York: The Free Press.

Halverson, Marvin y Cohen (Ed) (1962). A Handbook of Christian Theology. Essential Information for every Christian. Great Britain: Collins (Fontana Books).

\section{Bibliografía secundaria}

Cohn-Sherbok, Dan. (1997). Fifty Key Jewish Thinkers. London: Routledge.

Cronin, G. y Berger, A. (Ed) (2009). Encyclopaedia of Jewish-American Literature. New York: Facts on File Inc.

Giménez Blunden, M. (2012). Arthur Allen Cohen y el Tremendum. Una aproximación teológica a la Shoah. Revista El Olivo, 75, 87-106.

Mendes-Flohr, P. y Stern, D. (1998). An Arthur A. Cohen Reader. Selected fiction and writings on Judaism, theology, literature and culture. Detroit: Wayne State University Press.

Morgan, M. (2001). Beyond Auschwitz. Post-Holocaust Jewish Thought in America. Oxford University Press. 
Shatzky, J. y Taub, M. (Ed.) (1997). Contemporary Jewish-American Novelists. A BioCritical Sourcebook. London: Westport-Connecticut, Greenwood Press.

Rubenstein, R. (1996). After Auschwitz. History, Theology and Contemporary Judaism. Baltimore and London: The Johns Hopkins University Press. 